\title{
ADDENDUM
}

\section{Bright spatially coherent synchrotron X-rays from a table-top source}

S. Kneip, C. McGuffey, J. L. Martins, S. F. Martins, C. Bellei, V. Chvykov, F. Dollar, R. Fonseca, C. Huntington, G. Kalintchenko, A. Maksimchuk, S. P. D. Mangles, T. Matsuoka, S. R. Nagel, C. A. J. Palmer, J. Schreiber, K. Ta Phuoc, A. G. R. Thomas, V. Yanovsky, L. O. Silva, K. Krushelnick and Z. Najmudin

Nature Physics 6, 980-983 (2010); published online: 24 October 2010; corrected after print: 2 September 2011.

This paper presents the realization and comprehensive characterization of a compact bright source of ultrafast synchrotron radiation with appreciable degree of spatial coherence. It has been drawn to our attention that we had inadvertently neglected to cite some prior works that described selected aspects of the betatron radiation in similar experimental configurations: ref. 1 describes a direct source size measurement based on Fresnel diffraction from laser-based synchrotron radiation demonstrating the resolution that this measurement can yield, and refs 2 and 3 describe indirect measurements of the electron oscillation amplitude based on far-field analysis of the betatron profile and based on the linking of electron and X-ray spectra, respectively. The varied techniques of refs 1-3 give consistent answers for the X-ray source size. Refs 1-3 are consistent with our measurement and modelling. They underline the importance in our experiment to generate high-charge quasi-monoenergetic electron beams to scale up betatron energy and brightness whilst keeping the source size small.

1. Shah, R. C. et al. Coherence-based transverse measurement of synchrotron X-ray radiation from relativistic laser-plasma interaction and laser-accelerated electrons. Phys. Rev. E 74, 045401 (2006).

2. Phuoc, K. T. et al. Imaging electron trajectories in a laser-wakefield cavity using betatron X-ray radiation. Phys. Rev. Lett. 97, 225002, (2006).

3. Albert, F. et al. Betatron oscillations of electrons accelerated in laser wakefields characterized by spectral X-ray analysis. Phys. Rev. E 77, 056402 (2008). 